\title{
Revised Sections F7.5 (Quantitative Amino Acid Analysis) and F7.6 (Qualitative Amino Acid Analysis): American College of Medical Genetics Standards and Guidelines for Clinical Genetics Laboratories, 2003
}

Robert E. Grier, $P h D^{1-4,}$ William A. Gahl, MD, PhD ${ }^{2,5}$, Tina Cowan, PhD ${ }^{2,6}$, Isa Bernardini, $M S^{2,5}$, Geraldine A. McDowell, $P h D^{1,2,7}$ and Piero Rinaldo, $M D, P h D^{1-3,8}$

\begin{abstract}
Determination of plasma amino acid levels has become a key piece of information in the diagnosis and clinical management of a group of metabolic genetic disorders. Appropriate laboratory methodologies have been published for amino acid analysis, yet there is a need for direction for the laboratory in performing this testing. The following guidelines were generated by a working group of the American College of Medical Genetics Laboratory Quality Assurance Committee. Based upon a body of knowledge and professional experience, these guidelines and standards are to be the benchmark for performance of amino acid analysis for clinical interpretation. Genet Med
\end{abstract} 2004:6(1):66-68.

Key Words: metabolic disorders, amino acids, ion exchange chromatography

\section{F7.5 QUANTITATIVE AMINO ACID ANALYSIS}

\section{F7.5.1 Introduction}

The universal importance of amino acids stems from their role in literally all metabolic and cellular functions. Amino acids serve as protein building blocks, as metabolic intermediates, and as a source of energy. By definition, amino acids contain an amino group and a carboxyl group, and may contain another functional group (e.g., sulfhydryl, hydroxyl, or secondary amino or carboxyl group). Ion exchange chromatography analysis of amino acids in plasma is the most common diagnostic method to identify defects of amino acid metabolism. Screening methods such as thin layer chromatography that do not result in positive identification of all amino acids of clinical interest can lead to errors. Laboratories that use such methods should make the limitations of the methodology clear and indicate the appropriate follow-up testing in the patient report. The quantitative analysis of a clinical specimen requires three steps: (1) isolation/separation of the free amino acids from the physiological specimen; (2) separation and identification of the tagged or labeled compounds; and (3) quantitation of compounds by comparison to standards.

\footnotetext{
${ }^{1}$ ACMG Laboratory Quality Assurance Committee, ${ }^{2}$ ACMG Amino Acids Revision Working Group, ${ }^{3}$ ACMG Organic Acids Revision Working Group, ${ }^{4}$ Baylor University Medical Center, ${ }^{5}$ Heritable Disorders Branch, National Institute of Child Health and Human Development, ${ }^{6}$ University of Maryland at Baltimore, ${ }^{7}$ Laboratory Corporation of America, and ${ }^{8}$ Mayo Clinic.
}

American College of Medicine Genetics, 9650 Rockville Pike, Bethesda, MD 20814-3998.

See disclaimer at the end of this article.

DOI: 10.1097/01.GIM.0000106163.35058.7D

\section{F7.5.2 Background}

F7.5.2.1 Clinical description of disease (amino acidemias and acidurias): As a group of inborn errors of metabolism, amino acid defects are clinically and biochemically heterogeneous. They are characterized biochemically by the accumulation of pathological amounts of normal metabolites or by the accumulation of metabolites that are not present under physiological conditions but are produced from the activation of alternative pathways in response to the loss of function of a specific gene product (enzyme). The natural history of these disorders is variable and disease-specific.

F7.5.2.2 Prevalence: The birth of a child with an amino acid disorder is not rare. The estimated incidence of phenylketonuria (PKU) alone is $1: 12,000$, with the combined incidence for all amino acidopathies estimated at 1:6,000. This estimate does not include other inborn errors of metabolism (i.e., organic acid disorders, some urea cycle disorders, and congenital lactic acidemias) that may require amino acid analysis for diagnosis and monitoring of patient treatment.

F7.5.2.3 Mode of inheritance: The majority of amino acidopathies are inherited as autosomal recessive traits; a few are X-linked.

F7.5.2.4 Specimen requirements: Plasma is generally the most informative specimen type. Serum may be used but is less ideal. Serum samples are generally left to clot at room temperature, which can lead to artifacts from deamination, conversion of arginine to ornithine by red blood cell arginase, and release of oligopeptides. Urine amino acid analysis is necessary for the diagnosis of some disorders, primarily those involving defec- 
tive renal transport (e.g., cystinuria and renal Fanconi syndrome). Amino acid concentrations are more variable in urine than in plasma due to factors such as renal function and greater interference from medications. Therefore, urine amino acid analysis should be discouraged as a first tier investigation when screening for an inborn error of metabolism unless a specific disorder is suspected for which plasma is not informative or urine analysis is valuable for the purpose of differential diagnosis. Urine collection should avoid fecal contamination. Urine should be collected without preservatives. Cerebral spinal fluid (CSF) is useful in the diagnosis of several disorders, most notably nonketotic hyperglycinemia. CSF samples are most informative when a plasma sample is collected at the same time and the ratios of amino acid concentrations in CSF to plasma are calculated.

F7.5.2.5 Specimen processing: Once collected, timely centrifugation and separation of plasma or serum specimens is important to reduce the influence of other blood constituents on the soluble amino acids. Hemolysis should be avoided because red blood cells contain high levels of certain amino acids. Before analysis, the plasma or serum specimens must be refrigerated for the short term $(<4 \mathrm{hr})$ or frozen $\left(-20^{\circ} \mathrm{C}\right)$ to arrest amino acid degradation. CSF and urine must be frozen immediately to prevent loss of some amino acids. Note that free homocysteine levels in plasma specimens will be reduced over time as homocysteine binds to albumin and other plasma proteins. With timely processing and analysis of specimens, ion exchange chromatography is suitable for detecting the large elevations of homocystine found in homocystinuria. This method is not suitable for detecting moderate elevations of total homocysteine found in hyperhomocystinemia.

F7.5.2.6 Clinical indications for testing: The clinical presentations of different disorders of amino acid metabolism are variable and often nonspecific. Onset of symptoms may occur in the neonatal period or as late as adulthood. Amino acid analysis should be considered in many clinical situations, including when any of the following are present:

1. lethargy, coma, seizures, or vomiting in a neonate

2. hyperammonemia

3. ketosis

4. metabolic acidosis or lactic academia

5. alkalosis

6. metabolic decompensation

7. unexplained developmental delay

8. polyuria, polydipsia, and dehydration

9. abnormal amino acid results by newborn screening

10. previous sibling with similar clinical presentation

11. clinical presentation specific to an amino acid disorder

\section{F7.5.3 Guidelines}

F7.5.3.1 Preanalytical variables: Treatment of patients with antibiotics such as ampicillin or medications such as cough syrup and some anticonvulsants; nutritional status; and bacterial contamination of specimens may affect results and should be taken into account during interpretation.
F7.5.3.2 Specimen preparation: Deproteinization of the physiological specimen is necessary. A common laboratory method of specimen preparation is acidification of a known specimen volume with a known small volume of concentrated acid, such as sulfosalicylic acid or trichloroacetic acid (TCA) to precipitate proteins and large molecules, followed by centrifugation, leaving the water soluble amino acids in the supernatant for analysis. Another method is to use low molecular weight cut-off filtration. At least one internal standard is added to the specimen and the $\mathrm{pH}$ is monitored and adjusted if necessary. Several methods also utilize a microfiltration of the supernatant using a syringe/filter apparatus to protect the column and permit optimum chromatography.

F7.5.3.3 Separation techniques: Ion exchange chromatography is the most common method of amino acid separation and analysis. Other methods that result in the positive identification of amino acids, such as tandem mass spectrometry, may be used. Tandem mass spectrometry typically is used to measure specific amino acids for newborn screening.

When separating by ion exchange chromatography, derivatization of amino acids is required for detection and can be accomplished either precolumn with o-phthalaldehyde (OPA) or phenylisothiocyanate (PITC) or postcolumn using ninhydrin. Postcolumn ninhydrin derivatization is preferred because it involves minimal sample processing and produces more consistent results. Several autosampler/ion exchange/detector configurations are commercially available that allow for positive identification of amino acids by their chromatographic retention time. These instruments have published methodologies that must be validated in the individual laboratory.

F7.5.3.4 Calibration: Quantitation without the use of a reference standard is not acceptable. Quantitation should be based on the performance and regular updating of calibration curves covering the normal range and expected pathological values. The laboratory should determine the linearity of all clinically informative amino acids.

F7.5.3.5 Chromatogram analysis: Identification of amino acids by ion exchange chromatography relies primarily on chromatographic retention time and retention time relative to an internal standard. If ninhydrin is used for detection, the 440 to $570 \mathrm{~nm}$ ratio can be informative for identification of amino acids. Quantitation should be based on the recovery of the internal standard in each specimen compared to the recovery of the internal standard in the quality control amino acid mixture for each run (see F7.5.3.6).

F7.5.3.6 Quality control: A known control amino acid mixture should be analyzed repeatedly to establish an acceptable range for each analyte. This mixture should be analyzed periodically to verify the instrument. The frequency with which these standards are analyzed depends on the stability and use rate of the derivatization material. If patient samples are run in daily batches, the standard mix should be analyzed with each batch. The internal standard(s) in each specimen serves as a quality control (QC) check for each specimen. A QC program based on the quantitative analysis of normal and abnormal control specimens should be implemented on a regular basis. 
The use of Westgard rules for clinical specimen analysis further controls the parameters for quality patient diagnosis and reporting (Westgard and Klee, 1999).

F7.5.3.7 Interpretation and reporting: Patient and specimen information, as contained in Sections C2.4, C2.4.1, and C2.4.2 of the Standards and Guidelines for Clinical Genetics Laboratories, must be included on each report. The phone number of the reporting laboratory is required in case the referring physician has questions. Identification of all relevant amino acids should be listed and the quantity may be listed. When no significant abnormalities are detected, an amino acid analysis could be reported and interpreted in qualitative terms only. When abnormal results are detected, a detailed interpretation should include an overview of the results and their significance, a correlation to available clinical information, elements of a differential diagnosis, and recommendations for additional biochemical testing, including in vitro confirmatory studies (enzyme assay, molecular analysis). It must be recognized that reference values of several amino acids are characteristically age-dependent, thereby requiring that quantitative results be compared to a properly defined age group.

Whenever possible, confirmation of a diagnosis of an amino acid disorder by an independent method, typically by in vitro enzyme assay (blood cells, cultured cells, and tissue biopsy) or molecular analysis, is recommended. Interpretations of amino acid results are based upon relative amino acid levels, pattern recognition, and correlation of positive and negative findings, rather than on individual abnormal levels alone. Amino acid elevation(s) or overall profiles should be evaluated in the context of clinical findings and/or additional test results.

\section{F7.5.4 References and resources}

Blau N, Duran M, Blaskovics ME. Physician's guide to the laboratory diagnosis of metabolic diseases. New York: Chapman \& Hall Medical, 1996.
Bremer HJ, Duran M, Kamerling JP, Przyrembel H, Wadman SK. Disturbances of amino acid metabolism: Clinical chemistry and diagnosis. Baltimore-Munich: Urban \& Schwarzenberg, 1981.

Nyhan WL. Abnormalities in amino acid metabolism in clinical medicine. Norwalk: Appleton-Century-Crofts, 1984.

Slocum RH, Cummings JG. Amino acid analysis of physiological samples. In: Hommes FA, editor. Techniques in diagnostic human biochemical genetics. New York: Wiley-Liss, 1991:87-126.

Westgard JO, Klee GG. Quality management. In: Burtis CA, Ashwood ER, editors. Tietz textbook of clinical chemistry. Philadelphia: W.B. Saunders, 1999;384-419.

\section{F7.6 QUALITATIVE AMINO ACID ANALYSIS}

Qualitative amino acid analysis by thin layer chromatography (TLC) is suitable only for the detection of gross abnormalities. As some disorders may be missed by this method, its use for the purpose of evaluating high-risk patients should be discouraged. Qualitative amino acid analysis must reliably detect conditions in which there are either gross or modest elevations of specific amino acids in blood and/or urine.

\section{F7.6.1}

Reports should indicate the method used. If TLC was the method used, a statement should be added indicating that TLC is suitable only for detection of gross abnormalities, and that quantitative analysis of plasma, urine, or CSF using a more sensitive method is recommended for the diagnosis and monitoring of treatment of conditions characterized by abnormal amino acids in blood and/or urine.

Disclaimer: Go to www.geneticsinmedicine.org for a printable copy of this document.

This guideline is designed primarily as an educational resource for medical geneticists and other health care providers to help them provide quality medical genetic services. Adherence to this guideline does not necessarily assure a successful medical outcome. This guideline should not be considered inclusive of all proper procedures and tests or exclusive of other procedures and tests that are reasonably directed to obtaining the same results. In determining the propriety of any specific procedure or test, the geneticist should apply his or her own professional judgment to the specific clinical circumstances presented by the individual patient or specimen. It may be prudent, however, to document in the patient's record the rationale for any significant deviation from this guideline. 